\title{
Variación en el contenido de glomalina relacionada a las proteínas del suelo, después de un incendio forestal en un Andisol en bosques de Araucaria araucana del centro-sur de Chile
}

\author{
Change in total glomalin content related to soil proteins after a wildfire in an Andisol \\ of Araucaria araucana forests of south-central Chile
}

\author{
Yessica Rivas ${ }^{a *}$, María Inés Canseco ${ }^{\mathrm{b}}$, Heike Knicker ${ }^{\mathrm{c}}$ Paulina Etcheverría ${ }^{\mathrm{d}}$, Roberto Godoy ${ }^{\mathrm{e}}$, \\ Francisco Matus $^{f}$, Eduardo Valenzuela ${ }^{g}$, Roberto Gallardo ${ }^{\text {h }}$ \\ *Autor de correspondencia: ${ }^{a}$ Universidad de Concepción, Facultad de Ingeniería Agrícola, \\ Departamento de Recursos Hídricos, sede Chillán, Chile, tel.: 56-42-2208981, rivastisnao@gmail.com \\ ${ }^{\mathrm{b}}$ Universidad de la Frontera, Facultad de Ciencias Forestales y Agropecuarias, Temuco, Chile.

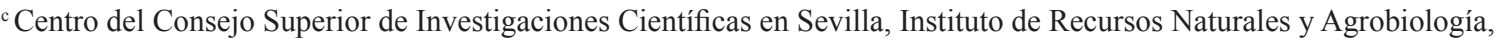 \\ E-41080 Sevilla, España. \\ ${ }^{d}$ Instituto Nacional de Investigación Agropecuaria (INIA) Quillamapu, Vicente Méndez 515, Chillán, Chile. \\ ${ }^{\mathrm{e}}$ Universidad Austral de Chile, Facultad de Ciencias, Instituto de Ciencias Ambientales y Evolutivas, Valdivia, Chile. \\ ${ }^{\mathrm{f}}$ Universidad de La Frontera, Departamento de Ciencias Químicas y Recursos Naturales, \\ Scientific and Technological Bioresource Nucleus (BIOREN-UFRO), Temuco, Chile. \\ ${ }^{g}$ Universidad Austral de Chile, Facultad de Ciencias, Instituto de Bioquímica y Microbiología, Valdivia, Chile. \\ ${ }^{\text {h } U n i v e r s i d a d ~ A u s t r a l ~ d e ~ C h i l e, ~ F a c u l t a d ~ d e ~ C i e n c i a s ~ F o r e s t a l e s ~ y ~ R e c u r s o s ~ N a t u r a l e s, ~ V a l d i v i a, ~ C h i l e . ~}$
}

\begin{abstract}
SUMMARY
In high-altitude montane forests, the vascular plants undergo mycorrhizal symbiosis with mycorrhizal fungi as a strategy to face extreme soil and climate conditions. In this study, glomalin content related to soil proteins (GRSP) was studied, an insoluble glycoprotein produced by hyphae of arbuscular mycorrhizal fungi and deposited on the soil, four years after a wildfire of variable severity, in Araucaria araucana forest in south-central Chile $\left(38^{\circ} \mathrm{S}\right)$. For the previously stated, the aim proposed was to determine the content of this glycoprotein as well as to evaluate and relate the composition of soil organic matter to the content of this glycoprotein. Samples were collected at different depths (0-5, 5-10, 10-20 and 20-30 cm, respectively) from areas presenting various fire severities (low, medium and high) and an unburned soil area (control soil). Furthermore, composition and structure of soil organic matter was studied by Nuclear Magnetic Resonance Spectroscopy of ${ }^{13} \mathrm{C}$ (NMR ${ }^{13} \mathrm{C}$ ). As main results, total GRSP concentration showed significant differences between the burned soil and the control soil. A significant correlation between the composition of the organic matter and GRSP was found. The high concentration of GRSP obtained in burned forests could answer to an ecological-evolutionary strategy from the Araucaria araucana forest in their adaptation to both a soil with low nutrient availability and to periodical fire catastrophic events; suggesting their key role in the recovery of these ecosystems and should be considered in restauration programs.
\end{abstract}

Key words: fire- glomalin- temperate forest- ${ }^{13} \mathrm{C}$ NMR spectroscopy.

\section{RESUMEN}

En bosques de alta montaña, las plantas vasculares establecen simbiosis con hongos micorrícicos como una estrategia a condiciones edafo-climáticas extremas. En este estudio, se analizó el contenido de glomalina relacionada a las proteínas del suelo (GRSP), una glicoproteína producida por hongos micorrícicos arbusculares y depositada en el suelo, después de cuatro años de ocurrido un incendio de severidad variable en un bosque de Araucaria araucana en el Centro-Sur de Chile ( $\left.38^{\circ} \mathrm{S}\right)$. Para lo anterior, se propuso como objetivo determinar el contenido de esta glicoproteína en el suelo así como evaluar y relacionar la composición de la materia orgánica con el contenido de esta. Se analizó muestras de suelo a distintas profundidades (0-5, 5-10, 10-20 y 20-30 cm, respectivamente) en sectores con diversos niveles de severidad de incendio (baja, media, alta) y un área no afectada por el incendio (control). Adicionalmente, se estudió la estructura y composición de la materia orgánica mediante espectrometría de resonancia magnética nuclear del ${ }^{13} \mathrm{C}\left({ }^{13} \mathrm{C} R \mathrm{RMN}\right)$. Como principales resultados, la concentración de GRSP mostró diferencias significativas entre el suelo quemado y suelo control. Se encontró una significativa correlación entre la composición de la materia orgánica y GRSP. La alta concentración de GRSP en los bosques quemados, podría responder a una estrategia ecológico-evolutiva de los bosques de $A$. araucana en su adaptación a suelos con limitantes nutricionales y sometidos a eventos catastróficos periódicos de incendios, que sugieren su rol clave en la recuperación de estos ecosistemas y deberían ser considerados en programas de restauración.

Palabras clave: fuego, GRSP, bosque templado, espectroscopia $\mathrm{RMN}{ }^{13} \mathrm{C}$. 


\section{INTRODUCCIÓN}

Los bosques templados lluviosos del Cono Sur de América representan ecosistemas únicos de gran valor ecológico y social. Además, proporcionan una gran variedad de bienes y servicios que son de enorme importancia medio ambiental y económica, tanto a nivel nacional como internacional (Armesto et al. 2009). Las recientes proyecciones del clima no solo indican que se acentúa el calentamiento global, sino también el incremento e intensidad de eventos climáticos extremos, con fuertes cambios en temperatura y precipitación, con una dinámica regional inusual (Gutiérrez et al. 2014). Estos cambios climáticos extremos tienen potencial para alteraciones rápidas y profundas de la biota y de los procesos biogeoquímicos en el ecosistema. La eficiencia en el ciclo de nutrientes y el rol de los microorganismos del suelo, son fundamentales para el mantenimiento de la productividad y estabilidad en estos ecosistemas (Huygens et al. 2008). Sin embargo, perturbaciones como los incendios catastróficos podrían tener un fuerte impacto en el balance de nutrientes en estos ecosistemas y consecuentemente, su capacidad de resiliencia puede ser amenazada (Perakis et al. 2002).

Los suelos donde han evolucionado esos ecosistemas boscosos de la Cordillera de Los Andes, son provenientes de cenizas volcánicas, caracterizados por contener bajas concentraciones de nitrógeno inorgánico (Huygens et al. 2008). Bajo este escenario de restricción nutricional los bosques templados han desarrollado estrategias funcionales compensatorias, aprovechando los recursos bióticos y abióticos del ecosistema. Entre ellas se destacan las asociaciones simbióticas con hongos micorrícicos arbusculares, que permiten suplir gran parte de estas deficiencias nutritivas en estos ecosistemas (Castillo et al. 2006, Smith y Read 2008).

Los hongos micorrícicos arbusculares se caracterizan por producir glomalina, una glicoproteína de gran adhesividad, insoluble en agua y de gran estabilidad a la temperatura y a la degradación enzimática (Wright y Upadhyaya 1996). La fracción de glomalina que se obtiene desde el suelo, ha sido definida operacionalmente como glomalina relacionada a las proteínas del suelo (GRSP, por sus siglas en inglés), dada la imposibilidad de separarla in vivo del resto de las proteínas del suelo (Nichols y Wright 2004, Rillig 2004a). La glomalina se produce durante la colonización de las raíces y la ramificación del micelio de hongos micorrícicos arbusculares al explorar el suelo (Rillig 2004b), y es incorporada directamente al suelo mediante la descomposición y transformación de los propágulos fúngicos (Schindler et al. 2007). La GRSP contiene una gran cantidad de proteínas relacionadas con el suelo y de origen no micorrícico (Gillespie et al. 2011). De acuerdo a Gillespie et al (2011), el procedimiento Bradford, utilizado en el presente estudio, sobrestima la cantidad de glomalina y hasta ahora, aún no se ha determinado en forma concluyente la naturaleza química de glomalina.
Esta macromolécula, dependiendo del tipo de vegetación y suelo, fluctúa normalmente entre 10 y $30 \%$ del contenido total del carbono del suelo (Nichols y Wright 2004, Rillig 2004a), llegando incluso a representar hasta un $52 \%$ del carbono total en suelos orgánicos (Schindler et al. 2007), lo que se ha descrito igualmente en la simbiosis micorrícicos arbusculares con especies típicas del bosque lluvioso templado (Etcheverría et al. 2009). Estudios en suelos volcánicos chilenos, bajo distintas coberturas vegetales han reportado valores de glomalina cercanos al $19 \%$ del contenido de carbono total del suelo (Etcheverría et al. 2009).

En el Parque Nacional Tolhuaca (región de La Araucanía), Cordillera de Los Andes, Centro-Sur de Chile, un incendio de gran magnitud (febrero del 2002) afectó a bosques prístinos de $A$. araucana ubicados en áreas remotas. El incendio creó un mosaico de severidades de afectación en el paisaje (\% de árboles muertos), observándose en una superficie superior a las 3.800 ha. En el presente trabajo se postula que el fuego disminuye la concentración de GRSP del suelo, observable después de cuatro años de ocurrido el incendio, como consecuencia de la pérdida de especies hospederas de hongos simbiontes, reducción del volumen radical y, en consecuencia, una disminución en las estructuras micorrízicas en el suelo.

Los objetivos de la presente investigación son: i) determinar el contenido de GRSP en el suelo y, ii) evaluar y relacionar la composición de la materia orgánica del suelo con el contenido de GRSP en el perfil del suelo, en bosques de $A$. araucana afectados por distintas severidades de incendio. Como control, se utilizó un área boscosa de formación similar pero no afectada por el fuego a lo menos en los últimos 150 años.

\section{MÉTODOS}

Área de estudio y colecta de suelos. El área de estudio comprendió diversos sectores del Parque Nacional Tolhuaca, ubicado entre 900 y 1.700 m s.n.m. en la Cordillera de Los Andes ( $38^{\circ} 10^{\prime}-38^{\circ} 15^{\prime} \mathrm{S}$ y $\left.71^{\circ} 41^{\prime}-71^{\circ} 50^{\prime} \mathrm{O}\right)$. Alrededor del $60 \%$ de la superficie del parque (aprox. 3.800 hectáreas) fue afectada por un incendio de gran magnitud entre enero y febrero del año 2002. La topografía del terreno es ondulada, 5-10\% de pendiente, exposición sur. El clima es de tipo templado lluvioso, con elevadas precipitaciones en invierno y sequía estival en verano (Amigo y Ramírez 1998). Las precipitaciones varían entre 1.500 y $2.500 \mathrm{~mm}$ anuales y la temperatura media del mes más frío (julio) es de $7,0^{\circ} \mathrm{C}$, mientras que la del mes más cálido (enero) es de $19,9^{\circ} \mathrm{C}$. Hay ocurrencias de nevadas entre julio y septiembre. Los suelos son considerados incipientes, formados sobre cenizas volcánicas recientes, muy estratificados, con areniscas incluidas y sin desarrollo de fuertes estructuras (Peralta 1976).

La vegetación dominante corresponde a comunidades boscosas con estrato arbóreo dominado por Araucaria 
araucana (Mol.) C. Koch, acompañada de Nothofagus spp. en estratos bajos del bosque. La regeneración del bosque, después de cuatro años de ocurrido el incendio, se ha realizado principalmente a través de semillas y rebrotes vegetativos adventicios de individuos de A. araucana que sobrevivieron al fuego. El establecimiento de plántulas de A. araucana proviene de semilla, principalmente en las áreas de baja severidad, al igual que de rebrotes de raíces. Otras especies arbóreas como Nothofagus nervosa (Phil.) Dim. et Mil. y Nothofagus antarctica (G. Forster) Oerst., han rebrotado exitosamente, en condiciones de baja y media severidad. Por otro lado, independiente de la severidad del incendio, Chusquea culeou Desv., se estableció más rápida y densamente, alcanzando una mayor cobertura e importancia que cualquiera de las otras especies en el sotobosque, junto a la especie herbácea geófita Alstroemeria aurea Graham, cabe mencionar que ambas especies se asocian con micorrizas del tipo arbuscular (Godoy et al. 1994). Por otro lado, ambas especies no colonizaron en aquellas áreas de mayor severidad del fuego. Si lo hicieron algunas especies herbáceas exóticas, debido aparentemente a la menor respuesta y cobertura de C. culeou, así como perturbación producto del tránsito de animales vacunos que ingresan al área.

Para el muestreo, en las distintas áreas de estudio, fueron seleccionadas parcelas permanentes elegidas al azar, después de cuatro años de ocurrido el evento de incendio.
En el área afectada por el fuego se eligieron distintos grados de severidad, definida como una medida del daño en base al porcentaje de árboles vivos post incendio, estableciendo las siguientes categorías: i) severidad alta $(<20 \%$ árboles vivos), ii) severidad media (20 - 60\%) y iii) severidad baja (60 - $80 \%$ ), además de un área control no afectada por fuego al menos en los últimos 150 años (González et al. 2005) (figura 1). Se estableció tres parcelas $(0,1 \mathrm{ha})$ para cada severidad y sitio no afectado por el fuego. Al centro de cada repetición se excavó una calicata de $0.3 \mathrm{~m}$ de profundidad, donde se colectaron muestras de suelo con un cilindro ( $5 \mathrm{~cm}$ de diámetro $\mathrm{x} 5 \mathrm{~cm}$ de largo) a los $0-5 ; 5-10 ; 10-20 \mathrm{y}$ 20-30 $\mathrm{cm}$ de profundidad, respectivamente. En total, se colectaron 48 muestras de suelo para los análisis respectivos. Las muestras de suelo fueron transportadas inmediatamente al laboratorio, donde se retiró los restos de materiales orgánicos visibles. Los suelos fueron secados al aire hasta humedad constante y posteriormente tamizados a $2 \mathrm{~mm}$.

Determinación de carbono y nitrógeno. La concentración de carbono total y nitrógeno total en el suelo fue determinada en duplicado mediante analizador elemental, por combustión seca a $950^{\circ} \mathrm{C}$. La concentración de carbono y nitrógeno total varió de un $12 \%$ a $18 \%$ y un $0,5 \%$ a $0,8 \%$, en los primeros $30 \mathrm{~cm}$ de profundidad del suelo, respectivamente. $\mathrm{El} \mathrm{pH}$ al agua de determinó en una relación suelo-solución 1:2.5. El pH del suelo es ácido, variando

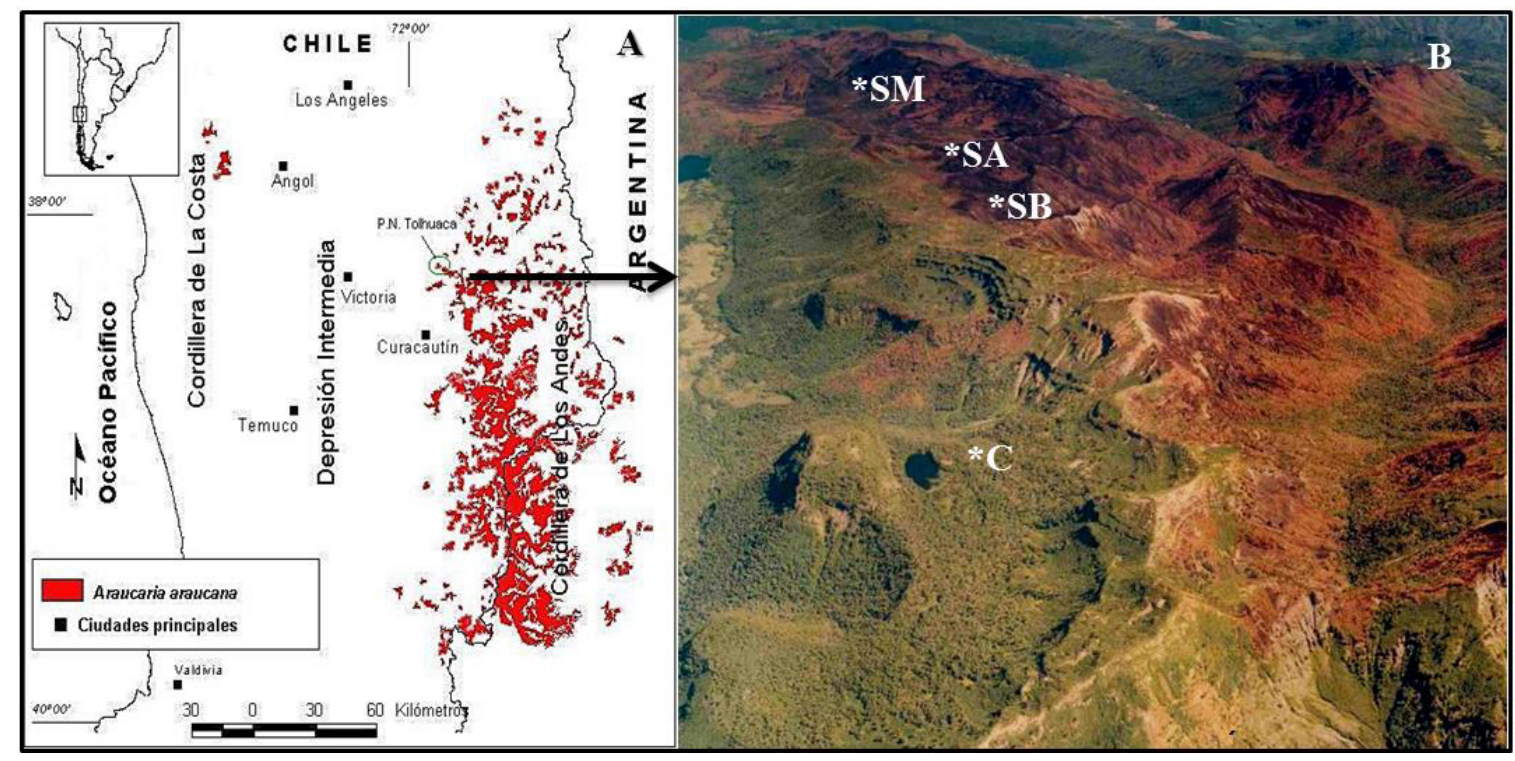

Figura 1. Área de distribución natural de la especie Araucaria araucana. El circulo detalla la ubicación del área de estudio: Parque Nacional Tolhuaca, centro-sur de Chile (A). Fotografía aérea año 2002 post-incendio (B). *Se señalan las áreas de estudio con distintas severidades de incendio estudiadas en el Parque Nacional Tolhuaca: SA: Severidad alta (<20 \% árboles vivos); SM: Severidad media (20-60 \% árboles vivos), SB: Severidad baja (60-80\% árboles vivos) y C: Bosque no afectado por el fuego (Control). (Fuente: CONAF 2002).

Natural distribution area of Araucaria araucana; in green location of study area: Tolhuaca National Park, South-Central Chile (Source: CONAF) (A). Aerial potographs post-fire 2002 (B) *that show the different wildfire severities studied in the Tolhuaca Nacional Park: High fire severity $(<20 \%$ of living trees), Medium fire severity (20-60\% of living trees), Low fire severity (60-80\% of living trees), and Unburned forest soil (control). (Source: CONAF 2002). 
entre 4,9 a 5,4 a la misma profundidad (mayor información en Rivas et al. 2012a).

Extracción y cuantificación de GRSP. La concentración de GRSP se determinó utilizando la metodología propuesta por Wright y Upadhyaya (1996). Para esto, el procedimiento de extracción de GRSP consistió en ciclos sucesivos en autoclave $\left(121^{\circ} \mathrm{C}\right)$ por 60 minutos, usando buffer citrato $50 \mathrm{mM}$ a pH 8,0 hasta que el sobrenadante no presentó color (aproximadamente cinco ciclos). El sobrenadante fue almacenado a $4{ }^{\circ} \mathrm{C}$, hasta su análisis. La cuantificación de GRSP total se realizó con la prueba de medición de proteínas totales de Bradford. Para ello se determinó la concentración de proteína total en el sobrenadante obtenido luego de la extracción con citrato de sodio, utilizando una curva estándar de albúmina. Los resultados fueron expresados como gramos de GRSP por kilogramo de suelo.

Resonancia magnética nuclear $d e l{ }^{13} \mathrm{C}$. Muestras de suelo obtenidas según se describió previamente, fueron sometidas a un tratamiento con ácido fluorhídrico para eliminar el material paramagnético (óxidos de hierro) y concentrar la Materia orgánica del suelo previo al análisis de resonancia nuclear del ${ }^{13} \mathrm{C}\left(\mathrm{RMN}{ }^{13} \mathrm{C}\right)$ en estado sólido, de acuerdo con el protocolo descrito por Knicker et al. (2006). Entre 110 y $275 \mathrm{mg}$ de muestra de suelo tratada fueron posteriormente oxidadas (6 horas) con $40 \mathrm{ml}$ de una solución de dicromato de potasio $\left(0,1 \mathrm{M} \mathrm{K}_{2} \mathrm{CrO}_{7} / 2,0 \mathrm{M} \mathrm{H}_{2} \mathrm{SO}_{4}\right)$ a $60{ }^{\circ} \mathrm{C}$ en un baño ultrasónico (Knicker et al. 2007). La oxidación de las muestras fue realizada en duplicado y la pérdida de carbono (C) de las muestras de suelo fue calculada respecto a muestras no oxidadas. Los residuos oxidados fueron lavados cuatro veces con agua destilada y posteriormente liofilizadas. Las muestras oxidadas fueron sometidas a análisis de resonancia nuclear del ${ }^{13} \mathrm{C}$ en estado sólido (espectrómetro Bruker DSX 200, Alemania), operando a una frecuencia de resonancia de ${ }^{13} \mathrm{C}$ de $50,3 \mathrm{MHz}$, usando rotores de dióxido de circonio de $7 \mathrm{~mm}$ de diámetro externo, con tapón KEL-F. La técnica de polarización cruzada se aplicó durante la rotación del ángulo mágico a $6,8 \mathrm{kHz}$. El tiempo de contacto fue de $1 \mathrm{~ms}$ y el intervalo entre pulsos de $1 \mathrm{~Hz}$ de $5,3 \mu \mathrm{s}$ a $90^{\circ}$. Se acumuló entre 3.000 y 10.000 escáneres, usando un pulso de retardo de $400 \mathrm{~ms}$. El desplazamiento químico se calibró respecto al estándar tetrametilsilano, usando glicina (COOH: 176,08 ppm). La cuantificación de las estructuras químicas determinadas se realizó mediante la división del espectro en diferentes regiones de desplazamiento químico: región $\mathrm{C}$-alquílica (0-45 ppm), región O-alquílica (45-110 ppm), región aromática (110-160 ppm) y región carboxílica (160-245). Las distintas señales fueron integradas usando los resultados del programa en forma relativa, de acuerdo a Knicker et al. (2005). Las bandas laterales de rotación originadas a ambos lados del patrón, como consecuencia de las condiciones de velocidad de rotación $(6,8 \mathrm{kHz})$ se corrigieron por contener un porcentaje de intensidad de las respectivas señales patrón. Así, a la intensidad de la región aromática (110-160 ppm) se le añadió la intensidad de las regiones 245-276 ppm y 0-45 ppm y a la región carboxílica (160245 ppm) se le añadió el doble de la señal de la banda lateral detectada a 276-323 ppm, menos la intensidad de la región alquílica (0-45 ppm) (Knicker et al. 2005).

Análisis estadístico. Se realizó un análisis de varianza de dos vías (ANDEVA de dos vías) para determinar la significancia del efecto de los factores: severidad del fuego y profundidad del suelo, sobre la concentración de GRSP, previa comprobación de la normalidad y homocedasticidad de la varianza. La comparación de medias entre severidades se realizó mediante la prueba de rango múltiple de Tukey $(P<0,05)$. Se determinaron correlaciones (Pearson) entre la concentración de GRSP y los contenidos de carbono total, nitrógeno total y los diferentes grupos funcionales asociados a la materia orgánica del suelo obtenido a través de resonancia magnética nuclear del ${ }^{13} \mathrm{C}$. $\mathrm{El}$ análisis estadístico fue realizado utilizando el paquete estadístico Statistics para Windows 6.0.

\section{RESULTADOS}

Contenido de GRSP y análisis elemental. No se encontró efecto significativo de la interacción de los factores severidad de incendio y profundidad de suelo sobre el contenido de GRSP. Sólo la severidad de incendio tuvo un efecto $(P<0,001)$. Los contenidos de GRSP en los suelos afectados por las distintas severidades de incendio variaron desde $32 \mathrm{~g} \mathrm{~kg}^{-1}$ a $41,3 \mathrm{~g} \mathrm{~kg}^{-1}$, a lo largo del perfil del suelo. No hubo diferencias significativas entre la concentración de GRSP y las distintas profundidades y severidades estudiadas (figura 2). En cambio en el suelo no afectado por el fuego, la concentración de GRSP fue mayor y fluctuó entre $42 \mathrm{~g} \mathrm{~kg}^{-1}$ y $57 \mathrm{~g} \mathrm{~kg}^{-1}$. Sin embargo, solo fue significativa esta diferencia entre los 5-10 cm de profundidad (figura 2).

Se encontró una positiva y significativa correlación entre GRSP y carbono total $(\mathrm{r}=0,79 ; P<0,001)$ a lo largo del perfil del suelo, en todas las parcelas estudiadas. De acuerdo a estos resultados, la concentración de carbono en el suelo es explicada en un $63 \%$ por el contenido de GRSP (figura 3). Por otro lado, la correlación entre GRSP y nitrógeno total (figura no incluida) fue baja pero significativa a lo largo de todo el perfil del suelo $(\mathrm{r}=0,633 ; P=0,009)$.

En relación a la composición de la materia orgánica del suelo, los resultados muestran un patrón común de acuerdo a la severidad del incendio (cuadro 1). Suelos expuestos a una mayor severidad del fuego presentaron una mayor participación de grupos aromáticos (lignina, 'black carbon', y otros similares). Por el contrario, aquellos suelos expuestos a una menor severidad del fuego, mostraron una disminución en la participación de aquellos grupos más aromáticos y un aumento en la concentración de los grupos C-alquílico (ceras, lípidos, entre otros), principalmente en los primeros $10 \mathrm{~cm}$ de profundidad (mayor información en Rivas et al. 2012a). 
Se encontró, principalmente hasta los $20 \mathrm{~cm}$ de profundidad, una negativa y significativa correlación entre la concentración de aquellos grupos más aromáticos (lignina, 'black carbon', y otros) de la materia orgánica del suelo y la concentración de GRSP (cuadro 2). Así a una mayor participación de los grupos más recalcitrantes de la materia orgánica, menor fue la concentración de GRSP encontrada. Por el contrario, hubo una positiva y significativa correlación entre la participación de aquellos grupos más hidrofóbicos de la materia orgánica del suelo, como C-alquílico (ceras, lípidos y otros) y GRSP total, principalmente entre los $5-10 \mathrm{~cm}$ de profundidad del suelo. Para otras profundidades, si bien fue alta correlación entre GRSP y el grupo alquílico, esta fue no significativa $(P>0,05)$.

\section{DISCUSIÓN}

Áreas remotas de montaña en el cono sur de América constituyen puntos de control privilegiados para realizar estudios de línea base, que permitan responder diversas in-
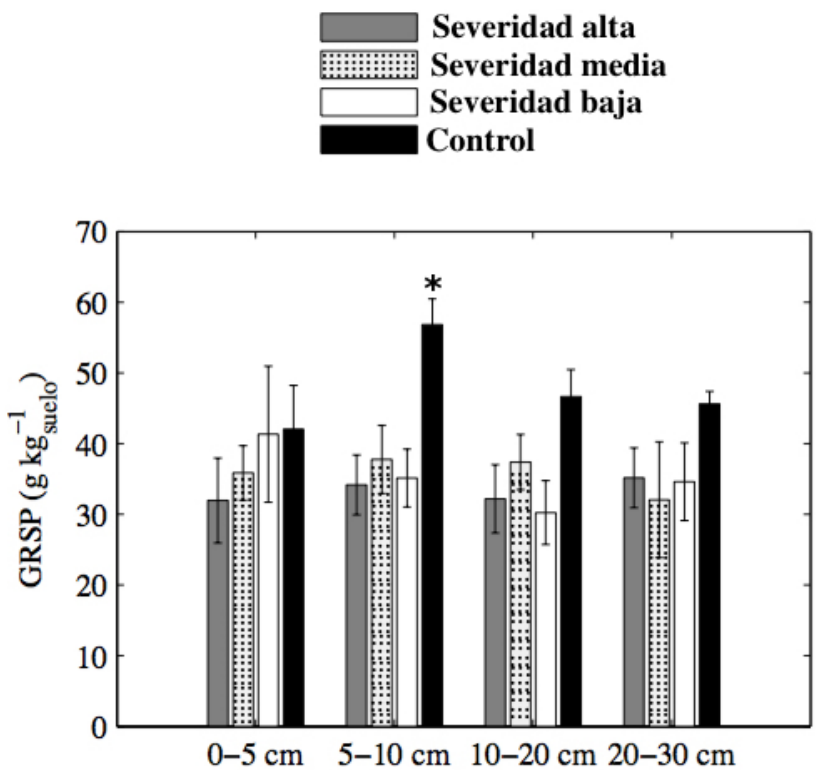

Figura 2. Contenidos de GRSP total ( $\mathrm{g} \mathrm{kg}^{-1}$ de suelo) (media \pm desviación estándar, $\mathrm{n}=9$ ), hasta los $30 \mathrm{~cm}$ de profundidad, para las siguientes severidades de incendio: severidad alta $(<20 \%$ árboles vivos), severidad media (20-60\% árboles vivos), severidad baja (60-80\% árboles vivos) y un área no afectada por fuego (control), en un Andisol con bosques de Araucaria araucana, en el Parque Nacional Tolhuaca (Centro-Sur de Chile). Diferencias significativas entre el suelo control y las distintas severidades estudiadas se indican con un asterisco* $(P<0,05)$.

Soil content of total glomalin-related to the soil protein (GRSP) ( $\mathrm{g} \mathrm{kg}^{-1}$ soil) until $30 \mathrm{~cm}$ of depth in various severities of forest fire: high fire severity ( $<20 \%$ of living trees), medium fire severity (20-60\% of living trees), low fire severity (60-80\% of living trees), and unburned forest soil (control) in an Andisol under Araucaria araucana forest stands from South-Central Chile. (Standard deviation in bars, $n=9$, significant differences between control and burned sites are indicated by $* P<0.05$ ). terrogantes ecológicas para modelar y proyectar potenciales efectos, frente a escenarios de perturbación catastrófica y cambio climático global. Periodos de sequía cada vez más prolongados para el Centro-Sur de Chile y disminución de las precipitaciones, aumentan la recurrencia de incendios, afectando la estabilidad de ecosistemas boscosos templados en la región (Armesto et al. 2009, Gutiérrez et al. 2014).

En el presente estudio, el fuego produjo en primera instancia una disminución en la densidad de las especies de plantas micorrícicas hospederas y cambios en las propiedades físico químico de la materia orgánica del suelo, principalmente en los primeros $10 \mathrm{~cm}$ (Knicker et al. 2007b, Rivas et al. 2012b). La pérdida de especies hospederas pudo afectar la concentración de GRSP total en el suelo, variando de acuerdo a la severidad del incendio. De acuerdo a los resultados obtenidos y después de cuatro años post-incendio, se encontró que si existe un efecto del fuego sobre la concentración de GRSP total, pero esta diferencia se produce principalmente entre los 5-10 cm de profundidad del suelo, sub-superficie, que además presenta los mayores contenidos de GRSP en el Andisol estudiado. La mayor cantidad de GRSP encontrada a esta profundidad coincide donde se concentra la mayor densidad radical, provenientes de especies de sotobosque y

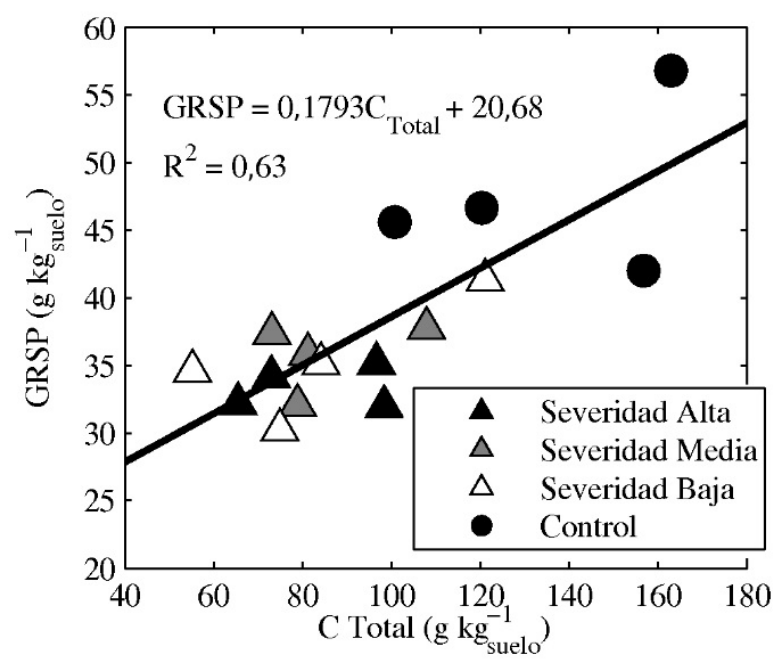

Figura 3. Correlación entre GRSP ( $\mathrm{g} \mathrm{kg}^{-1}$ de suelo) y carbono total ( $\mathrm{g} \mathrm{kg}^{-1}$ de suelo), hasta los $30 \mathrm{~cm}$ de profundidad, para las severidades de incendio: severidad alta $(<20 \%$ árboles vivos), severidad media (20-60 \% árboles vivos), severidad baja (60-80 $\%$ árboles vivos) y un área no afectada por el fuego (control), en un Andisol con bosques de Araucaria araucana, en el Parque Nacional Tolhuaca (Centro-Sur de Chile).

Relationship between total glomalin-related soil protein (GRSP) ( $\mathrm{g} \mathrm{kg}^{-1}$ soil) and total carbon ( $\mathrm{g} \mathrm{kg}^{-1}$ soil), until $30 \mathrm{~cm}$ of depth, in various severities of fire: high fire severity $(<20 \%$ of living trees), medium fire severity (20-60\% of living trees), low fire severity (60-80 $\%$ of living trees), and unburned forest soil (control) in an Andisol under Araucaria araucana forest stands from South-Central Chile. 
herbáceas asociadas, donde la gran mayoría de las plantas genera simbiosis del tipo arbuscular.

En relación a la densidad y distribución vertical de raíces, en plántulas de Nothofagus alpina asociada a ectomicorrizas en áreas quemadas y no quemadas en el mismo sector (Palfner et al. 2008), se observó que plántulas germinadas en suelo quemado presentaron una mayor presencia de raíces principales y muy baja o nula formación de raíces secundarias, en comparación a plántulas germinadas en un área no afectada por el fuego, principalmente en los primeros $10 \mathrm{~cm}$ de profundidad. Esta adaptación morfológica de las plántulas del área quemada podría estar asociada, a algunos factores abióticos como la baja disponibilidad de agua producto de un aumento en la hidrofobicidad, cambios en las propiedades físico-químico de la materia orgánica del suelo y otros efectos importantes del fuego sobre el suelo. Principalmente, sobre los primeros horizontes del suelo (Knicker et al. 2007b, Rivas et al., 2012a). Es importante destacar el establecimiento rápido y abundante de la especie herbáceas Alstroemeria sp. y la especie arbustiva C. culeou en las áreas afectadas por una severidad media y baja del fuego. Ambas especies realizan simbiosis con micorrizas del tipo arbuscular (Godoy et al. 1994), lo cual ayudó posiblemente a una rápida colonización radicular y recuperación de los niveles de GRSP total en el suelo, principalmente en los primeros horizontes del suelo. En esta misma línea, estudios realizados por Paulino (2006) en la misma área de estudio, encontraron que el 90 \% de las asociaciones micorrízicas de las plantas vasculares en bosques de Araucaria araucana, corresponden a asociaciones del tipo arbuscular, donde las especies de micorrizas del tipo arbuscular de los géneros Archaeospora, Acaulospora y Gigospora, fueron las únicas que resistieron el fuego en este ambiente, manteniendo su frecuencia en relación al área control. Los bosques templados del sur de Chile y Argentina, se caracterizan por una simbiosis en plantas vasculares con hongos arbusculares, más que asociaciones ectomicorrícicas, siendo estas últimas exclusivas del género Nothofagus (Godoy y Mayr 1989, Palfner et al. 2008). Sin embargo, aun cuando las ectomicorrizas no producen glomalina, es importante mencionar que estudios en Nothofagus spp., en la misma área de estudio afectada por incendios (Palfner et al. 2008), muestran que las plántulas de áreas quemadas presentan un menor porcentaje de micorrización y afectando la diversidad de especies de hongos ectomicorrízicos que colonizan después del paso del fuego. Esto corrobo-

Cuadro 1. Distribución de intensidad relativa (\%) de los principales constituyentes de la materia orgánica presentes en un suelo post-incendio en un bosque de Araucaria araucana del Centro- Sur de Chile a través de la integración de los espectros de resonancia magnética nuclear del ${ }^{13} \mathrm{C}$ en estado sólido.

Relative intensity distribution (\%) of the principal region of soil organic matter in a volcanic soil (Andisol) under Araucaria araucana forest stands from South-Central Chile, across solid-state ${ }^{13} \mathrm{C}$ nuclear magnetic resonance (NMR) spectra of the residue after hydrofluoric acid treatment and chemical oxidation resistant carbon.

\begin{tabular}{|c|c|c|c|c|c|}
\hline \multirow{3}{*}{ Severidad del fuego } & \multirow{3}{*}{$\begin{array}{l}\text { Profundidad } \\
\quad(\mathrm{cm})\end{array}$} & Carboxílico & C- Aromático & O-Alquil-C & C-Alquílico \\
\hline & & $(160-245 \mathrm{ppm})$ & $(110-160 \mathrm{ppm})$ & $(45-110 \mathrm{ppm})$ & $(0-45 \mathrm{ppm})$ \\
\hline & & \multicolumn{4}{|c|}{ - } \\
\hline \multirow{4}{*}{$\begin{array}{l}\text { Alta } \\
\text { ( }<20 \% \text { árboles vivos })\end{array}$} & $0-5$ & 12,1 & 46,5 & 7,1 & 34,4 \\
\hline & $5-10$ & 9,9 & 27,9 & 16,0 & 46,2 \\
\hline & $10-20$ & 9,7 & 13,9 & 5,6 & 70,9 \\
\hline & $20-30$ & 10,0 & 15,1 & 10,9 & 63,9 \\
\hline \multirow{4}{*}{$\begin{array}{l}\text { Media } \\
\text { (20-60 \% árboles vivos) }\end{array}$} & $0-5$ & 8,8 & 47,3 & 10,1 & 33,8 \\
\hline & $5-10$ & 13,4 & 31,7 & 8,1 & 46,8 \\
\hline & $10-20$ & 10,7 & 15,7 & 12,2 & 61,6 \\
\hline & $20-30$ & 9,8 & 13,5 & 12,4 & 64,3 \\
\hline \multirow{4}{*}{$\begin{array}{l}\text { Baja } \\
(60-80 \% \text { árboles vivos) }\end{array}$} & $0-5$ & 8,7 & 19,2 & 8,7 & 63,4 \\
\hline & $5-10$ & 11,9 & 14,3 & 10,7 & 63,1 \\
\hline & $10-20$ & 11,0 & 13,1 & 6,9 & 69,0 \\
\hline & $20-30$ & 15,1 & 17,2 & 6,0 & 61,9 \\
\hline \multirow{4}{*}{$\begin{array}{l}\text { Bosque no afectado por el fuego } \\
\text { (control) }\end{array}$} & $0-5$ & 7,0 & 6,0 & 13,1 & 73,9 \\
\hline & $5-10$ & 9,2 & 5,1 & 14,4 & 71,4 \\
\hline & $10-20$ & 10,0 & 4,0 & 14,3 & 71,7 \\
\hline & $20-30$ & 8,5 & 6,6 & 10,8 & 74,1 \\
\hline
\end{tabular}


raría la indudable dependencia que determinadas especies vegetales tienen de la micorrización en sus fases iniciales de desarrollo, incluso, tras un evento catastrófico como un incendio forestal, tanto a nivel de asociación endo- como ectomicorrízico. En este sentido, de acuerdo a los resultados presentados por Violi et al. (2008), tanto las asociaciones como las tasas de acumulación de GRSP en el suelo, se caracterizan por presentar gran resistencia y capacidad de resiliencia frente a distintos eventos catastróficos; como la deforestación, quemas controladas, entre otras. A pesar de lo anterior, los mismos autores señalan que la conversión de bosques maduros a plantaciones exóticas, cambia drásticamente la riqueza de especies de hongos arbusculares y la tasa de esporulación, pero no la tasa de producción de GRSP. Lo anterior también es mencionado por Paulino (2006), quién recalca que el fuego no produce un cambio en frecuencia en la micorrización si no que existe un cambio en la diversidad de especies micorrízicas que colonizan después del fuego. No obstante lo anterior, existen pocos estudios en ecosistemas boscosos donde se relacione un fenómeno de incendio de gran envergadura, sobre las asociaciones micorrízicas, más aun específicamente en el efecto del fuego sobre la concentración de GRSP en el suelo.
Respecto a la estructura química que presenta la fracción GRSP de la materia orgánica del suelo, estudios recientes revelan que es una mezcla de proteínas, ácidos húmicos, lípidos y sustancias inorgánicas (Gillespie et al. 2011), siendo aún poco clara su composición estructural. En el presente estudio, se encontró una fuerte y directa correlación entre la concentración de GRSP total, el carbono total del suelo y principalmente con los grupos funcionales alquílicos; compuestos generalmente por macromoléculas alifáticas tales como: ceras, lípidos, ácidos grasos, suberina, resina y diversas glicoproteínas, entre otros compuestos. En el estudio de Rivas et al. (2012a) se concluyó que los grupos alifáticos se oxidaron en menor grado que cualquier otro grupo funcional, enfatizando la importancia de la preservación de los grupos alquílicos en los sitios quemados respecto al área no afectada por el fuego, lo cual se corrobora con los altos niveles de GRSP encontrados en todos los sitios de este estudio. Por otro lado, se evidenció una correlación significativa e inversa entre la concentración de GRSP y los grupos aromáticos. La relación negativa con los grupos aromáticos de la materia orgánica del suelo, acentuada por el efecto de la severidad del fuego, muestra que los niveles de GRSP podrían ser afectados por

Cuadro 2. Coeficiente de correlación lineal de Pearson entre el contenido de GRSP total ( $\mathrm{g} \mathrm{kg}^{-1}$ suelo) y los distintos constituyentes de la materia orgánica del suelo (\%) post-incendio en un bosque de Araucaria araucana del Centro- Sur de Chile ( $\mathrm{n}=16)$. Diferencias significativas de las correlaciones, se indican con un asterisco $*<0,05$, n.s $=$ no significativo.

Correlation Pearson's coefficient between total glomalin-related soil protein (GRSP) and different regions of soil organic matter after wildfire under Araucaria araucana forest stands from South-Central Chile, after the treatment with hydrofluoric acid and chemical oxidation with potassium dichromate of $(\mathrm{n}=16)$. Significant differences between correlations are indicated by $* P<0.05, \mathrm{n} . \mathrm{s}=$ not significant $)$.

\begin{tabular}{cclc}
\hline Profundidad $(\mathrm{cm})$ & Severidad del fuego & Señal $(\mathrm{ppm})$ & Coeficiente de correlación lineal ( $\mathrm{r}$ de Pearson) \\
\hline \multirow{3}{*}{$0-5$} & SA & Carboxílico & $-0,11 \mathrm{n} . \mathrm{s}$ \\
& SM & C-Aromático & $-0,96^{* *}$ \\
& SB & O-Alquil-C & $0,46 \mathrm{n} . \mathrm{s}$ \\
& C & C-Alquílico & $0,87 \mathrm{n} . \mathrm{s}$ \\
\hline $5-10$ & SA & Carboxílico & $-0,90 \mathrm{n} . \mathrm{s}$ \\
& SM & C-Aromático & $-0,93^{* *}$ \\
& SB & O-Alquil-C & $0,01 \mathrm{n} . \mathrm{s}$ \\
& C & C-Alquílico & $0,99^{* *}$ \\
\hline \multirow{3}{*}{$10-20$} & SA & Carboxílico & $-0,51 \mathrm{n} . \mathrm{s}$ \\
& SM & C-Aromático & $-0,93^{* *}$ \\
& SB & O-Alquil-C & $0,06 \mathrm{n} . \mathrm{s}$ \\
& C & C-Alquílico & 0,85 \\
\hline \multirow{2}{*}{$20-30$} & SA & Carboxílico & $-0,56 \mathrm{n} . \mathrm{s}$ \\
& SM & C-Aromático & $-0,73 \mathrm{n} . \mathrm{s}$ \\
& SB & O-Alquil-C & $0,49 \mathrm{n} . \mathrm{s}$ \\
& C & C-Alquílico & $0,90 \mathrm{n} . \mathrm{s}$ \\
\hline
\end{tabular}

Severidad del fuego: SA: Severidad alta (<20\% árboles vivos); SM: Severidad media (20-60 \% árboles vivos), SB: Severidad baja (60-80 \% árboles vivos) y área no afectado por el fuego (control). 
la generación de productos más aromáticos como 'black carbon', la ciclación de componentes proteicos de la materia orgánica del suelo, la formación y acumulación de formas aromáticas de nitrógeno del tipo pirrol ('black nitrogen') (Knicker et al. 2007), lo cual podría explicar la baja correlación entre nitrógeno total del suelo y GRSP total. En general, los estudios sobre la naturaleza y composición de glomalina han sido limitados, debido a que se desconoce la naturaleza de esta proteínas y su relación específica con los hongos vesículo arbusculares (Gillespie et al. 2011). Sin embargo, Gadkar y Rilling (2006) aislaron e identificaron el gen (GiHsp 60) responsable de la síntesis de glomalina en los hongos vesículo arbusculares, identificando una secuencia de aminoácidos relacionadas a proteínas del estrés. Estas proteínas se activan y promueve una mayor producción de GRSP cuando disminuye la producción de micelio desde el hongo. Esta evidencia, nos ayuda a comprender porque la alta tasa de producción de GRSP se mantiene, aún después de eventos importantes de incendio.

Estas características de la GRSP, producto de las micorrizas arbusculares, podría responder a una estrategia ecológico-evolutiva de la simbiosis en este tipo particular de bosques templados, en su adaptación a condiciones edafo-climáticas restrictivas y a eventos catastróficos, como son los incendios naturales recurrentes, siendo claves en la recuperación y en potenciales programas futuros de restauración ecológica de ecosistemas degradados.

\section{CONCLUSIONES}

El contenido de GRSP en el suelo, como consecuencia de la actividad de los hongos micorrícicos arbusculares en sitios boscosos de alta montaña del Centro-Sur de Chile, es afectada significativamente por la ocurrencia del fuego, pero solo en los primeros horizontes del suelo.

Después de cuatro años de ocurrido un evento importante de incendio, existen tasas importantes de producción de GRSP en todas las áreas estudiadas. Esta singular característica que presentan los bosques de Araucaria araucana, puede representar una importante vía alternativa de recuperación, asociada a la capacidad de resiliencia del ecosistema.

La presente investigación entrega evidencias de que la fracción GRSP estaría asociada a la fracción más hidrofóbica de la materia orgánica del suelo, destacando además un efecto negativo en la producción de GRSP los compuestos más aromáticos productos del fuego.

Para trabajos futuros, sería muy interesante aislar y comparar aquellas micorrizas que colonizan actualmente en cada uno de las áreas de estudio, y proponerlo como alternativa a programas de restauración ecológica.

\section{AGRADECIMIENTOS}

Se agradece a: revisores anónimos por importantes contribuciones para mejorar versión final del manuscrito,
Proyecto FONDECYT Postdoctorado \# 3140161, al Dr. F. Borie por su apoyo en los análisis químicos en el Laboratorio de Ciencias Químicas, de la Universidad de la Frontera, Temuco; Proyecto DID-UACH (D-2006-17), Corporación Nacional Forestal (CONAF) y Guardaparques del Parque Nacional Tolhuaca, por su valiosa ayuda durante las campañas de muestreo.

\section{REFERENCIAS}

Amigo J, C Ramírez. 1998. A bioclimatic classification of Chile: woodland communities in the temperate zone. Plant Ecology 136(1): 9-26.

Armesto JJ, C Smith-Ramírez, M Carmona, JL Celis-Diez, I Díaz, A Gaxiola, AG Gutiérrez, M Núñez-Castillo CG, F Borie, R Godoy, R Rubio, E Sieverding. 2006. Diversity of arbuscular mycorrhizal plant species and fungal species in evergreen forest, deciduous forest and grassland ecosystems of Southern Chile. Journal of Applied Botany and Food Quality 80: 40-47.

Etcheverría P, D Huygens, R Godoy, F Borie, P Boeckx. 2009. Arbuscular mycorrhizal fungi contribute to ${ }^{13} \mathrm{C}$ and ${ }^{15} \mathrm{~N}$ enrichment of soil organic matter in forest soils. Soil Biology and Biochemistry 41(4): 858-861.

Gadkar V, M Rillig. 2006. The arbuscular mycorrhizal fungal protein glomalin is a putative homolog of heat shock protein 60. FEMS Microbiol Lett 263: 93-101.

Gillespie A, R Farrella, F Walley, A Ross, P Leinweber, K-U Eckhardt, T Regier, R Blyth. 2011. Glomalin-related soil protein contains non-mycorrhizal-related heat-stable proteins, lipids and humic materials. Soil Biology and Biochemistry 43(4): 766-777.

Godoy R, R Romero, R Carrillo. 1994. Estatus micotrófico de la flora vascular en bosques de coníferas del sur de Chile. Revista Chilena de Historia Natural 67(1): 209-220.

González M, T Veblen, J Sibold. 2005. Fire history of Araucaria-Nothofagus forests in Villarrica. National Park, Chile. Journal of Biogeography 32(7): 1187-1202.

Gutiérrez A, J Armesto, M Díaz, A Huth. 2014. Increased drought impacts on temperate rainforests from southern South America: results of a process-based, dynamic forest model. PLoS ONE 9(7): 1-15.

Huygens D, P Boeckx, P Templer, L Paulino, O Van Cleemput, C Oyarzún, C Müller, R Godoy. 2008. Mechanisms for retention of bioavailable nitrogen in volcanic rainforest soils. Nature Geoscience 1(8): 543-548.

Knicker H, F González-Vila, O Polvillo, J González, G Almendros. 2005. Fire induced transformation of C- and N- forms in different organic soil fractions from a Dystric Cambisol under a Mediterranean pine forest (Pinus pinaster). Soil Biology and Biochemistry 37(4): 701-718.

Knicker H, P Mueller, A Hilscher. 2007. How useful is chemical oxidation with dichromate for the determination of "Black Carbon" in fire-affected soils? Geoderma 142(1-2): 178-196.

Nichols KA, SF Wright. 2004. Contributions of soil fungi to organic matter in agricultural soils. In Magdoff F, R Weil eds. Functions and Management of Soil Organic Matter in Agroecosystems. Boca Ratón, Florida, USA. CRC Press. 179-198 p.

Palfner G, MI Canseco, A Casanova-Katny. 2008. Post-fire seed- 
lings of Nothofagus alpina in Southern Chile show strong dominance of a single ectomycorrhizal fungus and a vertical shift in root architecture. Plant and Soil 313(1-2): 237-250.

Paulino L. 2006. Efectos post-incendio sobre el ecosistema Araucaria-Nothofagus, Parque Nacional Tolhuaca. Tesis Doctorado en Ciencias, Mención Sistemática y Ecología. Valdivia, Chile. Universidad Austral de Chile. 186 p.

Peralta M. 1976. Uso, Clasificación y Conservación de Suelos. Santiago, Chile. Servicio Agrícola y Ganadero. 340 p.

Perakis S, L Hedin. 2002. Nitrogen loss from unpolluted South American forests mainly via disolved organic compounds. Nature 415(6870): 416-419.

Rillig M. 2004a. Arbuscular mycorrhizae and terrestrial ecosystem processes. Ecology Letters 7(8): 740-754.

Rillig M. 2004b. Arbuscular mycorrhizae, glomalin, and soil aggregation. Canadian Journal of Soil Science 84(4): 355-363.

Rivas Y, F Matus, H Knicker, C Rumpel, E Garrido. 2012a. Black carbon contribution in volcanic soils affected by wildfire or stubble burning Organic. Geochemistry 47: 41-50.

Rivas Y, D Huygens, H Knicker, R Godoy, F Matus, P Boeckx. 2012b. Soil nitrogen dynamics three years after a severe Araucaria-Nothofagus forest fire. Austral Ecology 37: 153-163.

Schindler FV, EJ Mercer, JA Rice. 2007. Chemical characteristics of glomalin-related soil protein (GRSP) extracted from soils of varying organic matter content. Soil Biology and Biochemistry 39(1): 320-329.

Smith SE, DJ Read. 2008. The roles of mycorrhizas in ecosystems. In Smith SE, DJ Read eds. Mycorrhizal Symbiosis. London, UK. Academic Press. p. 409-452.

Violi H, AF Barrientos-Priego, SF Wright, E Escamilla-Prado, JB Morton, JA Menge, C Lovatt. 2008. Disturbance changes arbuscular mycorrhizal fungal phenology and soil glomalin concentrations but not fungal spore composition in montane rainforests in Veracruz and Chiapas, Mexico. Forest Ecology and Management 254(2): 276-290.

Wright S, A Upadhyaya. 1996. Extraction of an abundant and unusual protein from soil and comparison with hyphal protein of arbuscular mycorrhizal fungi. Soil Science 161(9): 575-586.

Recibido: 21.10 .15

Aceptado: 14.04 .16 
